\title{
Alcune osservazioni sulle leges sumptuariae nel quadro delle campagne elettorali di età repubblicana
}

\author{
Lyuba Radulova
}

\author{
Università di Sofia "Sv. Kliment Ohridski" \\ Kontaktni e-mail: lyuba.radulova@gmail.com
}

\begin{abstract}
Some Observations on the leges sumptuariae in the Context of Electoral Campaigns in the Roman Republic
\end{abstract}

\begin{abstract}
:
The paper focuses on the Roman sumptuary legislation during the republican period, moving away from the traditional point of view which relates the leges sumptuariae to the crisis of the mores and the general decline of traditional values after the Punic wars. Instead, the key to understanding the sumptuary laws applied here is the euergetic activity, mainly in its practical aspects. The first part of the paper draws a clear distinction between the provisions limiting "personal luxury" and "euergetic luxury". The second part juxtaposes the sumptuary laws and the leges de ambitu, observing the mechanisms of direct electoral bribery and indirect political influence in the end of the Republic.
\end{abstract}

Keywords: Sumptus; banquets; ambitus; elections; campaigns; bribery

DOI: $10.14712 / 2464689 X .2020 .16$

Partendo dal concetto classico di "panem et circenses" il presente lavoro si propone di riesaminare la legislazione sumptuaria del periodo tardorepubblicano, scostandosi dall'ottica tradizionale della crisi dei mores e dei tentativi di ripristinare i valori tradizionali in conflitto con le influenze orientali. ${ }^{1}$ Come chiave di lettura, invece, viene applicata l'attività evergetica, considerata soprattutto nei suoi aspetti pratici. Si cerca in primo luogo, di distinguere tra le limitazioni del lusso "personale" e del lusso "evergetico". In secondo luogo, si osserva il parallelo funzionale e concettuale tra le leggi suntuarie e le leges de ambitu, concentrando l'attenzione sulla corruzione elettorale diretta e l'influenza politica indiretta, attuata attraverso atti evergetici.

Tra i principali studi dedicati alle leggi suntuarie andrebbero menzionati soprattutto quelli di SAUERWEIN, I. Die Leges sumptuariae als Römische Maßnahme gegen den Sittenverfall. Hamburg: Universität Hamburg, 1970; BALTRUSCH, E. Regimen morum: Die Reglamentierung des Privatlebens der Senatoren und Ritter in der römischen Republik und frühen Kaiserzeit. München: C. H. Beck, 1989 e BOTTIGLIERI, A. La legislazione sul lusso nella Roma repubblicana. Napoli: Edizioni scientifiche italiane, 2002. 


\section{Le proibizioni delle leggi suntuarie}

L'idea di limitare il lusso non è romana, ma si inserisce in una lunga tradizione di interventi greci in materia, attestata già a partire del VII-VI s. a.C. ${ }^{2}$ alla quale Platone dà anche uno sviluppo teorico. ${ }^{3}$ Queste leggi greche, pur non arrivando a una legislazione sul lusso esauriente ed organizzata, costituiscono un gruppo relativamente omogeneo di provvedimenti diretti contro qualsiasi ostentazione di lusso, nell'ambito funerario, ${ }^{4}$ nell'ambito cultuale e, spesso, nell'ambito dell'abbigliamento e dei gioielli femminili. ${ }^{5}$ Quanto ai provvedimenti contro il lusso a Roma, gli studiosi concordano che si tratta di un prestito concettuale greco, nell'adozione del quale hanno un ruolo decisivo Polibio e Catone. ${ }^{6}$

Meno univoca, invece, è l'interpretazione del concetto di lex sumptuaria. Alcuni studiosi preferiscono intendere il termine lex sumptuaria in senso largo, includendo tra le leggi suntuarie qualsiasi provvedimento diretto contro le spese eccessive e l'ostentamento di lusso, a prescindere se le fonti definiscano il detto provvedimento come lex sumptuaria

2 Sulla cronologia dell'apparizione dell'idea di limitazione del lusso in Grecia cfr. NENCI, G. Tryphé e colonizzazione. In: AA. VV. Modes de contacts et processus de transformation dans les sociétés anciennes. Actes du colloque de Cortone (24-30 mai 1981). Rome: Ecole française de Rome, 1983, pp. 1019-1031, soprattutto p. 1021. Sul concetto di tryphé cfr. PASSERINI, A. La tryphe nella storiografia ellenistica. Studi italiani di filologia classica, 1934, Vol. 11, pp. 35-56; COZZOLI, U. La tryphe nella interpretazione delle crisi politici. In: AA.VV. Tra Grecia e Roma. Temi antichi e metodologie moderne. Roma: Istituto della Enciclopedia italiana, 1980, pp. 133-145; LOMBARDO, M. Habrosune e habra nel mondo greco arcaico. In: AA. VV. Modes de contacts et processus de transformation dans les sociétés anciennes. Actes du colloque de Cortone (24-30 mai 1981). Rome: Ecole française de Rome, 1983, pp. 1077-1103; COUDRY, M. Loi et société: la singularité des lois somptuaires de Rome. Cahiers du Centre Gustave Glotz, 2004, Vol. 15, pp. 135-171, soprattutto pp. 135-139.

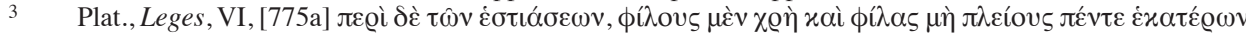

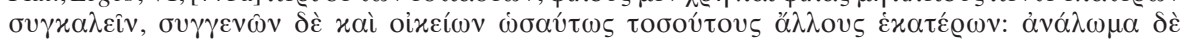

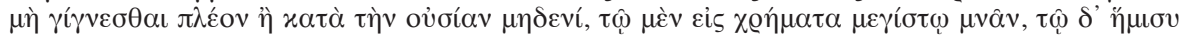

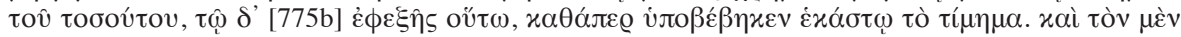

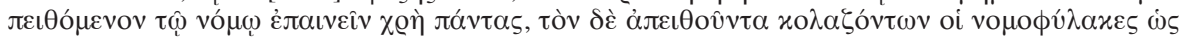

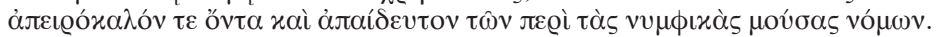

4 AMPOLO, C. Il lusso funerario e la città arcaica. AION, 1984, Vol. 6, pp. 71-102; FRISONE, F. Leggi e regolamenti funerari nel mondo greco, I. Le fonti epigrafiche. Lecce: Congedo, 2000 (con ampia bibliografia); COUDRY, Loi et société, p. 136.

5 MILLS, H. Greek Clothing Regulations: Sacred and Profane. ZPE, 1984, Vol. 55, pp. 255-265; COUDRY, Loi et société, p. 136.

6 PASSERINI, op . cit., p. 54; LETTA, C. L'Italia dei mores Romani nelle Origini di Catone. Athenaeum, 1984, Vol. 62, pp. 3-30; BONAMENTE, M. Leggi suntuarie e loro motivazioni In: AA.VV. Tra Grecia e Roma. Temi antichi e metodologie moderne. Roma: Istituto della Enciclopedia italiana, 1980, pp. 67-91; COUDRY, Loi et société, pp. 135-136. 
o meno. ${ }^{7}$ Così tra le leges sumptuariae ricadono anche i provvedimenti, diretti contro il lusso nell'abbigliamento ${ }^{8}$ o contro i regali troppo costosi. ${ }^{9}$

L'approccio minimalistico, ${ }^{10}$ invece, si basa su due opere letterarie che offrono elenchi dei provvedimenti definiti esplicitamente come leges sumptuariae - le opere di Aulo Gellio ${ }^{11}$ e di Macrobio. ${ }^{12}$ Vista la struttura ed il contenuto simili delle due fonti, gli studiosi fanno risalire entrambe ad un elenco modello preesistente, forse redatto dal giurista Ateio Capitone in età tiberiana. ${ }^{13}$ Questi elenchi contengono un numero minore di provvedimenti con tematica molto più omogenea e concentrata solo sul problema dei banchetti. ${ }^{14}$

Senza addentrarsi nel dibattito sul rapporto tra le leges sumptuariae di Gellio e Macrobio e le cosiddette leges cibariae, dedicate esclusivamente al lusso culinario, ${ }^{15}$ in questa sede si segue l'approccio minimalista, in quanto si condivide l'idea che nel definire come sumptuariae solo alcune delle leggi, dedicate al lusso, gli elenchi di Aulo Gellio e Macrobio abbiano compiuto una scelta informata, seguendo una logica valida, anche se forse diversa da quella di molti studiosi moderni.

7 BALTRUSCH, op. cit.; SAUERWEIN, op. cit.; BOTTIGLIERI, op. cit. Al tema specifico dell'interpretazione del termine lex sumptuaria è dedicato il contributo di KVASHNIN, V. Did Leges Sumptuariae exist in Rome? To Question About Correlation Terms Leges Sumptuariae and Leges Cibariae. Diritto@Storia.Rivista Internazionale di Scienze Giuridiche e Tradizione Romana, 2010, Vol. 9, [cit. 15. 11. 2019]. Available at: http://www.dirittoestoria.it/9/D\&Innovazione/Kvashnin-Leges-sumptuariae .htm.

8 Si tratta della Lex Metilia de fullonibus del 217 a.C. (Plin. NH 35, 191-202, cfr. BALTRUSCH, op. cit., pp. 50-52; VISHNIA R. F. Gaius Flaminius and the lex Metilia de Fullonibus. Athenaeum, 1987, Vol. 75, No. 29, pp. 527-534; WALLINGA, T. Official Roman Washing and Finishing Directions Lex Metilia Fullonibus Dicta. Revue d'Histoire du Droit, 1996, Vol. 64, No. 2, pp. 183-190) e la Lex Oppia del 215 a.C. (Liv. 34, 1, 3; Val. Max. 9, 1, 3; Tac. Ann. 3, 33, 4; Tac. Ann. 3, 34, 6; cfr. BALTRUSCH, op. cit., pp. 52-59; COUDRY, M. Loi Oppia limitant le luxe des femmes (pl. sc.). In: Lepor. Leges Populi Romani, sous la dir. de Jean-Louis Ferrary et de Philippe Moreau. [online]. 17. 11. 2019 [cit. 19. 11. 2019]. Paris: IRHT-TELMA, 2007. Available at: http://www.cn-telma.fr/lepor/notice570/.)

9 Cfr. Lex Publicia de cereis del 209 a.C. (Macr. Sat. 1, 7, 33; cfr. BALTRUSCH, op. cit., pp. 61-63) e Lex Cincia de donis et muneribus del 204 a.C. (Cic. Senect. 10, 4; BALTRUSCH, op . cit., pp. 63-69).

10 VISHNIA, op. cit., pp. 527-534; ROSIVACH, V. J. The Lex Fannia Sumptuaria of 161 B.C. CJ, 2006, Vol. 102, No. 1, pp. 1-15, soprattutto p. 1; CASINOS MORA, F. J. La restricción del lujo en la Roma Republicana. El lujo indumentario. Madrid: Dykinson, 2015, pp. 138-140.

11 Aul. Gell. Noct. Att. 2, 24; II. sec. d.C.

12 Macr. Sat., 3, 17; V. sec. d.C.

13 BALTRUSCH, op. cit., p. 77; COUDRY, Loi et société, p. 135-171; ROSIVACH, op. cit., p. 2. All'età di Tiberio risale anche l'ultimo trattamento in materia di legislazione suntuaria (in Tac. Ann. 2, 33; Tac. Ann. 3, 52-55. COUDRY, Loi et société, pp. 143-145).

14 Macr. Sat. 3, 17, 13: Cato enim sumptuarias leges cibarias appellat. Cfr. ROSIVACH, op. cit., pp. 1-15. Cfr. VISHNIA, op. cit., pp. 527-534; DE LIGT, L. Restraining the Rich, Protecting the Poor: Symbolic Aspects of Roman Legislation. In: JONGMAN, W. - KLEJWEGT, M. (eds.). After the Past. Essays in Ancient History in Honour of H. W. Pleket. Leiden: Brill, 2002, pp. 1-45, soprattutto pp. 3-4; DAUER, M. Roman Republican Sumptuary Legislation: 182-102 B.C. In: DEROUX, C. (ed.). Studies in Latin Literature and Roman History. Vol. 11. Bruxelles: Latomus, 2003, pp. 65-93. 
La più antica delle leggi elencate da Gellio e Macrobio è Lex Orchia sumptuaria ${ }^{16}$ del 182 a.C. Le fonti indicano che essa fissa un numero massimo dei partecipanti a un convivium ${ }^{17}$ e un valore massimo del banchetto ${ }^{18}$ pari a 100 assi. Una testimonianza di Festo ${ }^{19}$ parrebbe suggerire anche un divieto di usare schiavi per la distribuzione degli inviti al banchetto; tuttavia, l'attribuzione del testo di Festo alla lex Orchia non è universalmente accettata. ${ }^{20}$

Al 161 a.C. risale la Lex Fannia sumptuaria ${ }^{21}$ Essa viene preceduta da un senatusconsultum dello stesso anno, ${ }^{22}$ secondo il quale i principes civitatis dovrebbero giurare di non spendere più di 120 assi per i banchetti in occasione dei Ludi Megalenses, escludendo le spese per il holus, il far e il vino domestico; si tratta, dunque, di una limitazione del cibo di lusso comprato al mercato. Si propone di vietare, inoltre, l'introduzione al convivium di più di 100 libre di argento. La lex Fannia pare riprendere i provvedimenti del senatusconsultum, ${ }^{23}$ fissando, però, diversi valori massimi dei banchetti a seconda delle occasioni 100 assi per le maggiori feste, 30 assi per altri 10 giorni importanti al mese e 10 assi per i giorni feriali. ${ }^{24}$ A proposito di questi valori massimi, tuttavia, si ipotizza che ne vengano

16 Macr. Sat. 3, 17, 2-3 e 5; Fest., p. 220 L, s.v. obsonitauere; s.v. percunctatum; Schol. Bob., p. 14. ROTONDI, G. Leges publicae populi Romani. Hildesheim: Olms, 1966, p. 276; SAUERWEIN, op. cit., pp. 70-74; LINTOTT, A. Imperial expansion and moral decline in the Roman Republic. Historia, 1972, Vol. 21, pp. 626-638, soprattutto pp. 631-632; CLEMENTE, G. Le leggi sul lusso e la società romana tra il III e il II secolo a.C. In: GIARDINA, A. - SCHIAVONE, A. (eds.). Società romana e produzione schiavistica. III. Roma: Laterza, 1981, pp. 3-14, soprattutto p. 9; BALTRUSCH, op. cit., pp. 77-81; BOTTIGLIERI, op. cit., pp. 132-136; ELSTER, M. Die Gesetze der mittleren römischen Republik. Text und Kommentar. Darmstadt: Wissenschaftliche Buchgesellschaft, 2003, pp. 337-339; KVASHNIN, op cit.; COUDRY, Loi et société, pp. 138, 152. COUDRY, M. Loi Orchia somptuaire (pl. sc.). In: Lepor. Leges Populi Romani, sous la dir. de Jean-Louis Ferrary et de Philippe Moreau. [online]. 28. 11. 2014 [cit. 15. 11. 2019]. Paris: IRHT-TELMA, 2007. Available at: http://www.cn-telma.fr/lepor/notice571/.

17 Macr. Sat. 3, 17, 2: ... summa autem eius praescribebat numerum conuiuarum.

18 Fest., p. 220 L, s.v. obsonitauere: ... saepe obsonauere. Cato in suasione <ne > de lege Orchia derogaretur: "Qui antea obsonitauere, postea centenis obsonitauere».

19 Fest., p. 280 L, s.v. percunctatum: patris familiae nomen ne quis seruum mitteret, lege sanctum fuisse ait Cato in ea qua legem Orchiam <dis> suadet.

20 BALTRUSCH, op . cit., p. 79 riferisce la testimonianza di Festo direttamente alla lex Orchia, pensando a un uso di nomenclatores anche nell'organizzazione di banchetti. COUDRY, Loi Orchia, sottolinea che i nomenclatores in età repubblicana paiono essere collegati solo alla salutatio e alle campagne elettorali e, dunque, propone di riferire la nota di Festo a una lex de ambitu. Sul legame tra le leggi suntuarie e le leggi contro l'ambitus e sui tentativi di mettere sotto controllo lo sviluppo delle clientele, cfr. LINTOTT, Imperial Expansion, pp. 631-632; CLEMENTE, op. cit., pp. 3-14. COUDRY, Loi Orchia, invece, insiste che non bisogna osservare i provvedimenti della lex Orchia solo all'interno del contesto politico ed elettorale.

21 Plin. NH 10, 139; Aul. Gell. Noct. Att. 2, 24, 2-3; Aul. Gell. Noct. Att. 20, 1, 23; Athen. VI, 274 c-d; Macr. Sat. 3, 13, 13; Macr. Sat. 3, 16, 14-16; Macr. Sat. 3, 17, 3-5. ROTONDI, op . cit., pp. 287-288; SAUERWEIN, op. cit., pp. 78-89; BALTRUSCH, op. cit., pp. 81-85; GRUEN, E. Studies in Greek Culture and Roman Politics. Leiden - New York: Brill, 1990, pp. 172-173; BOTTIGLIERI, op. cit., pp. 136-148; ELSTER, op. cit., pp. 396-400; COUDRY, Loi et société, pp. 153-154; ROSIVACH, op. cit., pp. 1-15; COUDRY, M. Loi Fannia somptuaire. In: Lepor. Leges Populi Romani, sous la dir. de Jean-Louis Ferrary et de Philippe Moreau. [online]. 28. 11. 2014 [cit. 15. 11. 2019]. Paris: IRHT-TELMA, 2007. Available at: http://www.cn-telma.fr/lepor/notice380/.

22 Aul. Gell. Noct. Att. 2, 24, 2-3; ROSIVACH, op. cit., pp. 1-15; COUDRY, Loi Fannia somptuaire.

23 Sulla probabile inclusione dei provvedimenti del senatusconsultum anche nella lex Fannia cfr. ROSIVACH, op. cit., p. 4. Cfr. CASINOS MORA, op. cit., p. 193.

24 Aul. Gell. Noct. Att. 2, 24, 3: Sed post id senatus consultum lex Fannia lata est, quae ludis Romanis, item ludis plebeis et Saturnalibus et aliis quibusdam diebus in singulos dies centenos aeris insumi concessit decemque aliis diebus in singulis mensibus tricenos, ceteris autem diebus omnibus denos. A proposito dell'obiettivo del provvedimento di inserire i banchetti di ampio respire nella matrice fissa del calendario della comunità, cfr. ROSIVACH, op. cit., p. 4. 
esclusi i prodotti domestici, menzionati dal senatusconsultum. ${ }^{25} \mathrm{Si}$ conoscono alcuni limiti culinari specifici: al convivio non si può offrire più di una gallina a patto che non sia fatta ingrassare appositamente ${ }^{26}$ o un maiale farcito; ${ }^{27}$ c'è un limite alla carne affumicata. ${ }^{28}$ Ateneo, ${ }^{29}$ infine, parla di un limite massimo degli invitati, offrendo, tuttavia, numeri poco credibili: 3 ospiti nei giorni feriali e 5 nei giorni di mercato. È chiaro che le grandi festività prevedevano un numero di invitati molto maggiore.

La Lex Didia sumptuaria ${ }^{30}$ del 143 a.C. estende le sanzioni della lex Fannia non solo agli organizzatori, ma anche agli invitati. Alquanto problematica è la testimonianza, secondo la quale la validità della lex Fannia viene estesa universa Italia, comprendendo gli Italici. Il testo di Macrobio lascia il dubbio se si tratti dei cittadini romani che si trovino in Italia $^{31} \mathrm{o}$, invece, di Italici senza civitas Romana. ${ }^{32}$ Pare più convincente l'interpretazione secondo la quale oggetto della legge Didia siano i soli cittadini romani in Italia.

25 ROSIVACH, op. cit., pp. 1-15; questa possibilità non viene trattata da BALTRUSCH, op. cit., pp. 81-85 o COUDRY, Loi Fannia somptuaire.

26 Plin. NH 10, 139: Gallinas saginare Deliaci coepere, unde pestis exorta opimas aues et suopte corpore unctas deuorandi. Hoc primum antiquis cenarum interdictis exceptum inuenio iam lege Gai Fanni consulis undecim annis ante tertium Punicum bellum, ne quid uolucre poneretur praeter unam gallinam quae non esset altilis, quod deinde caput translatum per omnes leges ambulauit.

27 Macr. Sat. 3, 13, 13: Nam Titius in suasione legis Fanniae obicit saeculo suo quod porcum Troianum mensis inferant, quem illi ideo sic uocabant, quasi aliis inclusis animalibus grauidum, ut ille Troianus equus grauidus armatis fuit.

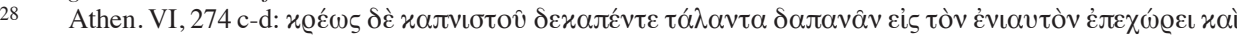

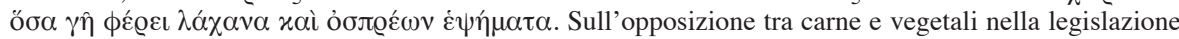
suntuaria cfr. COUDRY, Loi Fannia somptuaire. Sull'opposizione tra prodotti domestici e prodotti di lusso comprati al mercato e la logica economica e culturale dietro tale opposizione cfr. ROSIVACH, op. cit., pp. 6-7.

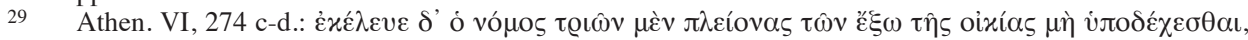

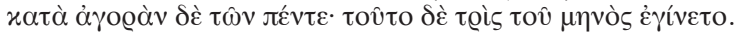

30 Macr. Sat. 3, 17, 6: Fanniam legem post annos decem et octo lex Didia consecuta est. Eius ferundae duplex fuit causa, prima et potissima ut uniuersa Italia, non solo urbs, lege sumptuaria teneretur, Italicis existimantibus Fanniam legem non in se sed in solos urbanos ciues esse conscriptam; deinde ut non soli qui prandia cenasue maiore sumptu fecissent, sed etiam qui ad eas uocitati essent atque omnino interfuissent, poenis legis tenerentur. ROTONDI, op. cit., p. 295; SAUERWEIN, op. cit., pp. 89-91; HARRIS, W. V. Was Roman Law Imposed on the Italian Allies? Historia, 1972, Vol. 21, pp. 639-645, soprattutto pp. 644-645; GALSTERER, H. Herrschaft und Verwaltung im republikanischen Italien: die Beziehungen Roms zu den italischen Gemeinden vom Latinerfrieden 338 v. Chr. bis zum Bundesgenossenkrieg 91 v. Chr. München: C. H. Beck, 1976, pp. 37-38, 132; BALTRUSCH, op . cit., pp. 85-86; BOTTIGLIERI, op. cit., p. 140; ELSTER, op. cit., pp. 434-435; COUDRY, M. Loi Didia somptuaire. In: Lepor. Leges Populi Romani, sous la dir. de Jean-Louis Ferrary et de Philippe Moreau. [online]. 23. 5. 2014 [cit. 15. 11. 2019]. Paris: IRHT-TELMA, 2007. Available at: http://www.cn-telma.fr/lepor/notice369/.

31 HARRIS, op. cit., pp. 644-645; GALSTERER, op. cit., pp. 37-38, 132; COUDRY, Loi Didia somptuaire.

32 SAUERWEIN, op. cit., pp. 89-91; GABBA, E. Rome and Italy in the Second Century BC. In: ASTIN, A. E. - WALBANK, F. W. - FREDERIKSEN, M. W. - OGILVIE, R. M. (eds.). The Cambridge Ancient History. 2nd ed. Vol. VIII. Rome and the Mediterranean to 133 BC. Cambridge: Cambridge University Press, 2008, pp. 197-243, soprattutto pp. 227-228. 
La Lex Licinia sumptuaria ${ }^{33}$ databile a un momento ignoto nel periodo tra il 143 e il 103 a.C. ${ }^{34}$ riprende in gran parte le disposizioni della lex Fannia. ${ }^{35}$ Vengono stabilite le spese massime per un banchetto, differenti per i giorni festivi, i giorni feriali e le nozze. ${ }^{36}$ Vengono definite anche quantità massime per certi prodotti alimentari di lusso. ${ }^{37}$ Come nella lex Fannia si osserva di nuovo la distinzione tra prodotti “domestici” e prodotti comprati al mercato che sono oggetto di limitazioni.

Nel 115 a.C. ${ }^{38}$ la Lex Aemilia sumptuaria aggiunge alle disposizioni della lex Licinia, ancora in vigore, ${ }^{39}$ limitazioni del tipo e della quantità di determinati alimenti di lusso uccelli esotici e molluschi. ${ }^{40}$

Nell'81 a.C. ${ }^{41}$ Silla restituisce le limitazioni al lusso di tavola attraverso la Lex Cornelia sumptuaria. Essa riproduce il modello delle lex Fannia e lex Licinia, fissando una spesa

33 Lucil. 1200 M (= H 54 C) Laevius; Fragm. Poet. Lat. 23; Aul. Gell. Noct. Att. 2, 24, 7; Aul. Gell. Noct. Att. 15, 8, lem.; Aul. Gell. Noct. Att. 20, 1, 23; Fest., p. 47 L, s.v. centenariae cenae; Macr. Sat. 3 , 17, 7-9; Val. Max. 2, 9, 5. Cfr. ROTONDI, op. cit., pp. 327-328; ASTE, G. Autore e tempo della lex Licinia de sumptu minuendo. Aevum, 1941, Vol. 15, pp. 581-588; SAUERWEIN, op. cit., pp. 94-113; CRAWFORD, M. Roman Republican Coinage. Cambridge: Cambridge University Press, 1974, pp. 624-625; BALTRUSCH, op. cit., pp. 88-93; RYAN, F. X. Favorinus, Favonius - and Sarmentus. Maia, 1994, Vol. 46, pp. 189-195; BOTTIGLIERI, op. cit., pp. 155-160; COUDRY, Loi et société, p. 154; COUDRY, M. Loi Licinia somptuaire. In: Lepor. Leges Populi Romani, sous la dir. de Jean-Louis Ferrary et de Philippe Moreau. [online]. 30. 1. 2019 [cit. 15. 11. 2019]. Paris: IRHT-TELMA, 2007. Available at: http://www.cn-telma.fr/lepor/notice510/.

34 Sulla datazione cfr. ASTE, op. cit., pp. 581-588; COUDRY, Loi Licinia somptuaire.

35 Macr. Sat. 3, 17, 8: Lex uero haec paucis mutatis in plerisque cum Fannia congruit. In ea enim ferenda quaesita est nouae legis auctoritas, exolescente metu legis antiquioris...

36 Le fonti offrono dettagli divergenti. Gellio attesta un limite di 100 assi per determinati giorni (le feste elencate dalla lex Fannia), 200 assi per le nozze e 30 per i giorni ordinari. Cfr. Aul. Gell. Noct. Att. 2, 24, 7: Lex deinde Licinia rogata est, quae cum certis diebus, sicuti Fannia, centenos aeris inpendi permisisset, nuptiis ducenos indulsit ceterisque diebus statuit aeris tricenos. Macrobio parla di due categorie di giorni, speciali (calende, none, idi) con un limite di 30 assi e ordinari con limitazione della quantità e del tipo di prodotti da offrire. Cfr. Macr. Sat. 3, 17, 9: Sed legis Liciniae summa ut Kalendis Nonis nundinis Romanis cuique in dies singulos triginta dumtaxat asses edundi causa consumere liceret, ceteris uero diebus, qui excepti non essent, ne amplius daretur apponeretur quam carnis aridae pondo tria et salsamentorum pondo libra et quod ex terra uite arboreue sit natum.

37 Ibid. Macr. Sat. 3, 17, 9: ... ceteris uero diebus, qui excepti non essent, ne amplius daretur apponeretur quam carnis aridae pondo tria et salsamentorum pondo libra et quod ex terra uite arboreue sit natum.

38 Plin. NH 8, 223; Aul. Gell. Noct. Att. 2, 24, 12; Macr. Sat. 3, 17, 13; Vir. ill. 72, 5. Cfr. ROTONDI, op. cit., p. 320; SAUERWEIN, op. cit., pp. 120-127; GABBA, E. Ricchezza e classe dirigente romana fra III e I sec. a.C. RSI, 1981, Vol. 93, pp. 541-558; BALTRUSCH, op. cit., pp. 86-88; BOTTIGLIERI, op. cit., pp. 165-166; COUDRY, Loi et société, pp. 155, 160; COUDRY, M. Loi Aemilia somptuaire. In: Lepor. Leges Populi Romani, sous la dir. de Jean-Louis Ferrary et de Philippe Moreau. [online]. 17. 11. 2019 [cit. 19. 11. 2019]. Paris: IRHT-TELMA, 2007. Available at: http://www.cn-telma.fr/lepor/notice14/.

39 La lex Licinia viene abrogata intorno al 97 a.C. dalla lex Duronia. Cfr. Val. Max. 2, 9, 5. ROTONDI, op. cit., pp. 334-335; SAUERWEIN, op. cit., pp. 96-97; BALTRUSCH, op. cit., p. 92; BOTTIGLIERI, op. cit., pp. 160-161. COUDRY, Loi et société, p. 161; COUDRY, M. Loi Duronia abrogeant la loi Licinia somptuaire (pl. sc.). In: Lepor. Leges Populi Romani, sous la dir. de Jean-Louis Ferrary et de Philippe Moreau. [online]. 23. 5. 2014 [cit. 15. 11. 2019]. Paris: IRHT-TELMA, 2007. Available at: http://www.cn-telma.fr/lepor/notice375/.

40 Plin. NH 8, 223: saurices et ipsos hieme condi auctor est Nigidius, sicut glires, quos censoriae leges princepsque $M$. Scaurus in consulatu non alio modo cenis ademere $<$ quam $>$ conch $<y>$ lia aut ex alio orbe conuectas aues. Aul. Gell. Noct. Att. 2, 24, 12: Praeter has leges Aemiliam quoque legem inuenimus, qua lege non sumptus cenarum, sed ciborum genus et modus praefinitus est. Macr. Sat. 3, 17, 13: Sulla mortuo Lepidus consul legem tulit et ipse cibariam - Cato enim sumptuarias leges cibarias appellat. Vir. ill. 72, 5: Consul legem de sumptibus et libertinorum suffragiis tulit.

41 Plut. Sull. 35, 2-3; Aul. Gell. Noct. Att. 2, 24, 11; Amm. 16, 5, 1; Macr. Sat. 3, 17, 11-12. Cfr. VOIGT, M. Über die lex Cornelia sumptuaria. BSGW, 1890, Vol. 42, pp. 244-290; ROTONDI, op. cit., pp. 354-355; CARCOPINO, J. Sylla ou la monarchie manquée. Paris: L'artisan du livre, 1931, pp. 57-60; SAUERWEIN, 
massima per un banchetto variabile a seconda dei giorni feriali o festivi. ${ }^{42}$ A ciò si aggiunge anche l'imposizione di un limite massimo dei prezzi di diversi generi alimentari di lusso. ${ }^{43}$ Di difficile interpretazione è la notizia di Plutarco ${ }^{44}$ secondo la quale Silla avrebbe imposto un limite anche alle spese per i funerali e poi l'avrebbe violato in occasione del funerale della moglie. Nonostante sia abbastanza chiaro che ci sia una notevole affinità concettuale tra la limitazione delle spese per i banchetti e quella delle spese per il funus, ${ }^{45}$ pare che esista una differenza teorica tra la legislazione sul lusso e la legislazione funeraria, essendo quest'ultima di ordine cultuale. ${ }^{46}$

Nel 68 a.C. una Lex Antia sumptuaria 47 introduce di nuovo limitazioni alle spese per i banchetti, anche se le fonti non offrono dettagli a proposito. ${ }^{48}$ Inoltre, la lex Antia

op. cit., pp. 130-140; HINARD, F. Sylla. Paris: Fayard, 1985, pp. 252-253; TOHER, M. The Tenth Table and the Conflict of Orders. In: RAAFLAUB, K. (ed.). Social Struggles in Archaic Rome. Berkeley - Los Angeles - London: Blackwell, 1986, pp. 301-326, soprattutto pp. 323-324; BALTRUSCH, op. cit., pp. 48-49 e 93-96; HANTOS, T. Res publica constituta. Die Verfassung des Dictators Sulla. Stuttgart: Franz Steiner Verlag, 1988, p. 66; FLOWER, H. I. Ancestor Masks and Aristocratic Power in Roman Culture. Oxford: Clarendon Press, 1996, p. 118; ENGELS, J. Funerum sepulcrorumque magnficentia: Begräbnisund Grabluxusgesetze in der griechisch-römischen Welt mit einigen Ausblicken auf Einschränkungen des funeralen und sepulkralen Luxus im Mittelalter und in der Neuzeit. Stuttgart: Franz Steiner Verlag, 1998, pp. 171-172; HINARD, F. Histoire romaine, I - Des origines à Auguste. Paris: Fayard, 2000, pp. 674, 678; BOTTIGLIERI, op. cit., pp. 162-165; WYETZNER, P. Sulla's Law on Prices and the Roman Definition of Luxury. In: AUBERT, J.-J. - SIRKS, A. J. B. (eds.). Speculum iuris. Roman Law as a Reflection of Social and Economic Life in Antiquity. Ann Arbor: University of Michigan Press, 2002, pp. 15-33; COUDRY, Loi et société, pp. 155-156, 159-160; COUDRY, M. Loi Cornelia sumptuaria. In: Lepor. Leges Populi Romani, sous la dir. de Jean-Louis Ferrary et de Philippe Moreau. [online]. 10. 10. 2018 [cit. 15. 11. 2019]. Paris: IRHT-TELMA, 2007. Available at: http://www.cn-telma.fr/lepor/notice163/.

42 Rispetto ai provvedimenti precedenti Silla aumenta il valore massimo, fissando 30 sesterzi per i giorni ordinari e 300 sesterzi per i giorni di feste o giochi. Cfr. Aul. Gell. Noct. Att. 2, 24, 11: Postea L. Sulla dictator, cum legibus istis situ atque senio oblitteratis plerique in patrimoniis amplis elluarentur et familiam pecuniamque suam prandiorum <conuiuiorum>que gurgitibus proluissent, legem ad populum tulit, qua cautum est, ut Kalendis, Idibus, Nonis diebusque ludorum et feriis quibusdam sollemnibus sestertios trecenos in cenam insumere ius potestasque esset, ceteris autem diebus omnibus non amplius tricenos.

43 Macr. Sat. 3, 17, 11-12: Has sequitur lex Cornelia et ipsa sumptuaria, quam tulit Cornelius Sulla dictator, in qua non conuiuiorum magnificentia prohibita est nec gulae modus factus, uerum minora pretia rebus imposita: et quibus rebus, di boni, quamque exquisitis et paene incognitis generibus deliciarum! Quos illic pisces quasque offulas nominat, et tamen pretia illis minora constituit! Ausim dicere ut uilitas edulium animos hominum ad parandas obsoniorum copias inpossent. Sulla logica economica dietro tale provvedimento cfr. CARCOPINO, op. cit., pp. 57-60; SAUERWEIN, op. cit., p. 134; BALTRUSCH, op. cit., p. 95; WYETZNER, op. cit., pp. 15-33.

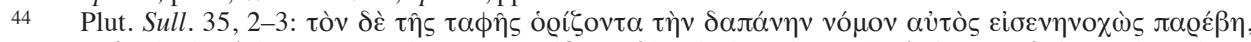

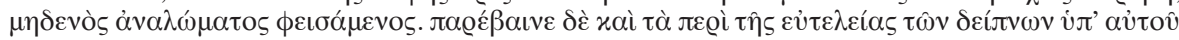

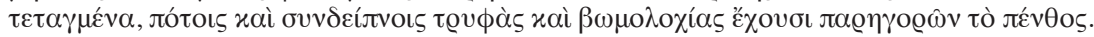
ENGELS, op. cit.; FLOWER, op. cit. Concorda COUDRY, Loi Cornelia sumptuaria.

TOHER, op. cit., pp. 301-326.

47 Aul. Gell. Noct. Att. 2, 24, 13; Macr. Sat. 3, 17, 13. Cfr. ROTONDI, op. cit., pp. 367-368; SYME, R. Ten Tribunes. JRS, 1963, Vol. 53, pp. 55-60; SAUERWEIN, op. cit., pp. 140-144; GRIFFIN, M. The Tribune C. Cornelius. JRS, 1973, Vol. 63, pp. 196-213; LINTOTT, Imperial Expansion, sopratutto pp. 5-6. BALTRUSCH, op. cit., pp. 96-98; NADIG, P. Ardet ambitus: Untersuchungen zum Phänomen der Wahlbestechungen in der römischen Republik. Frankfurt am Main: Peter Lang, 1997; BOTTIGLIERI, op. cit., pp. 166-167; COUDRY, Loi et société, p. 156; COUDRY, M. Loi Antia sumptuaria (pl. sc.). In: Lepor. Leges Populi Romani, sous la dir. de Jean-Louis Ferrary et de Philippe Moreau. [online]. 17. 11. 2019 [cit. 19. 11. 2019]. Paris: IRHT-TELMA, 2007. Available at: http://www.cn-telma.fr/lepor/notice28/. Aul. Gell. Noct. Att. 2, 24, 13: Lex deinde Antia praeter sumptum aeris id etiam sanxit, ut... 
proibisce $^{49}$ ai candidati per cariche magistratuali ${ }^{50}$ di partecipare più volte a banchetti se non da determinate persone. ${ }^{51}$

L'ultima legge suntuaria repubblicana, la Lex Iulia ${ }^{52}$ del 46 a.C. limita di nuovo le spese per i banchetti ${ }^{53}$ e vieta l'uso di determinati prodotti alimentari di lusso. ${ }^{54}$ Alcuni studiosi ${ }^{55}$ ipotizzano che la lex sumptuaria contenesse anche disposizioni riguardo il lusso femmini$1 \mathrm{e}^{56} \mathrm{e}$ il lusso funerario, ${ }^{57}$ anche se ciò non pare abbastanza convincente. ${ }^{58}$

La breve rassegna delle leges sumptuariae appena proposta potrebbe essere riassunta così:

1. Le leges, elencate da Gellio e Macrobio, sono concentrate esclusivamente sul lusso dei banchetti e, tranne pochissime eccezioni non trattano altri tipi di spese eccessive.

2. Si osservano tre gruppi di disposizioni:

49 Ibid. Aul. Gell. Noct. Att. 2, 24, 13: Lex deinde ... sanxit, ut qui magistratus esset magistratumue capturus esset, ne quo ad cenam, nisi ad certas personas, itaret.

50 La distinzione fatta tra magistratus e magistratum capturus pare identificare come destinatari del provvedimento sia i candidati, nel periodo delle campagne elettorali, sia i magistrati designati. Cfr. SAUERWEIN, op. cit., p. 144. Coudry (Loi Antia sumptuaria) ipotizza la possibilità che oggetto della lex Antia siano anche i magistrati in carica.

51 Probabilmente la legge contiene anche un elenco di tali personae certae, che forse sono legati al magistrato da diversi gradi di parentela. Cfr. MOREAU, Ph. La "Domus Augusta" et les formations de parenté à Rome. $C C G, 2005$, Vol. 16, pp. 7-23; COUDRY, Loi Antia sumptuaria.

52 Cic. Fam. 7, 26, 2; Cic. Fam. 9, 15, 5; Cic. Fam. 9, 26, 4; Cic. Att. 12, 13, 2; Cic. Att. 13, 7, 1; Suet. Iul. 43, 3; Dio 43, 25, 1-2. Cfr. ROTONDI, op. cit., p. 421; REINHOLD, M. History of Purple as a Status Symbol in Antiquity. Bruxelles: Latomus, 1970, pp. 45-46; SAUERWEIN, op. cit., pp. 147-156; JEHNE, M. Der Staat des Dictators Caesar. Köln: Böhlau, 1987, pp. 86-88; BALTRUSCH, op. cit., pp. 49-50, 59-60, 99-100; DERY, C. The lex sumptuaria in Cicero, Fam. 7, 26. Liverpool Classical Monthly, 1993, Vol. 18, No. 10, pp. 156-157; BOTTIGLIERI, op. cit., pp. 171-172; COUDRY, Loi et société, p. 162; COUDRY, M. Loi Iulia sumptuaria. In: Lepor. Leges Populi Romani, sous la dir. de Jean-Louis Ferrary et de Philippe Moreau. [online]. 9. 1. 2019 [cit. 15. 11. 2019]. Paris: IRHT-TELMA, 2007. Available at: http:// www.cn-telma.fr/lepor/notice481/.

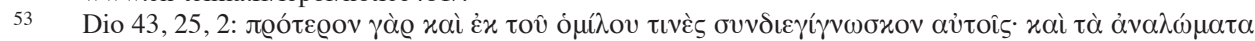

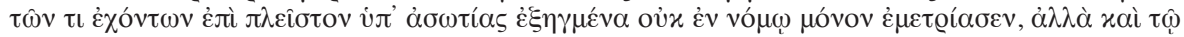

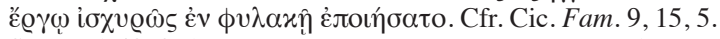

54 Suet. Iul. 43, 3: legem praecipue sumptuariam exercuit dispositis circa macellum custodibus, qui obsonia contra uetitum retinerent deportarentque ad se, submissis nonnumquam lictoribus atque militibus, qui, si qua custodes fefellissent, iam adposita e triclinio auferrent. Da notare che Cicerone informa che la legge vieta l'uso di pesce e frutti di mare, mentre non limita i vegetali (Cic. Fam. 7, 26, 2: Ac tamen, ne mirere

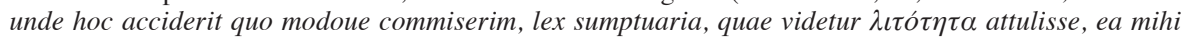
fraudi fuit. Nam dum uolunt isti lauti terra nata, quae lege excepta sunt, in honorem adducere, fungos, heluellas, herbas omnis ita condiunt ut nihil possit esse suauius. In eas cum incidissem in cena augurali

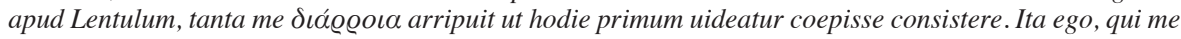
ostreis et murenis facile abstinebam, a beta et a malua deceptus sum.).

55 ROTONDI, op. cit., p. 421; JEHNE, op. cit., pp. 86-88.

56 Suet. Iul.43.2: Lecticarum usum, item conchyliatae vestis et margaritarum nisi certis personis et aetatibus perque certos dies ademit. Coudry (Loi Iulia sumptuaria) preferisce collegare tale provvedimento alle disposizioni riguardo la condotta delle donne o a quelle regolanti l'importazione di prodotti di lusso e non alla legislazione suntuaria (Suet. Iul. 43. 1; DE LAET, S. J. Portorium. Étude sur l'organisation douanière chez les Romains, surtout à l'époque du Haut-Empire. Bruges: De Tempel, 1949, pp. 60-61).

57 Cic. Att. 12, 35, 2; Cic. Att. 12, 36, 1. Cfr. BALTRUSCH, op. cit., pp. 49-50.

58 COUDRY, Loi Iulia sumptuaria. 
a) Limitazione della spesa massima per il banchetto; 59

b) Limitazione del numero degli invitati; 60

c) Limitazione del tipo e della quantità di cibi di lusso; ${ }^{61}$

\section{Leggi suntuarie e ambitus. Alcune osservazioni pratiche}

La prima osservazione che si potrebbe fare a proposito è che difficile identificare i modelli greci di questi provvedimenti. Come osserva Coudry, ${ }^{62}$ finora sono solo noti due provvedimenti, di $\operatorname{Iasos}^{63}$ e di Atene ${ }^{64}$ che limitano il numero degli invitati ai banchetti nuziali ed un ragionamento teorico in questo senso nelle Leggi di Platone ${ }^{65}$ sempre limitato solo ai banchetti nuziali. In più, solo il documento di Iasos indica anche la spesa massima per il banchetto. Pare, dunque, che i tre tipi di disposizioni delle leggi suntuarie romane siano un'innovazione romana, legata indubbiamente alle particolarità del modello culturale di Roma. ${ }^{66}$

La seconda osservazione che si può avanzare è che, vista la forte predominanza dell'interesse per la spesa massima e per il numero degli invitati, estranei alla domus, e, in più, il fatto che le limitazioni della spesa sono definite a base di un banchetto e non in relazione al numero degli invitati, pare che l'obiettivo principale delle leges sumptuariae sia quello di prevenire la riunione di un grande numero di invitati con un menù attraente. D'altronde, proprio l'offerta di un pasto, possibilmente desiderabile, a larghi gruppi di persone rappresenta uno dei più comuni atti evergetici. ${ }^{67}$ Pare, dunque, che le leges sumptuariae siano dirette non tanto contro il lusso culinario in ambito privato, quanto contro la dimensione evergetica e sociale del pasto collettivo.

È soprattutto questa importanza sociale (ma non solo) del dono del pasto che collega la legislazione suntuaria alle leges de ambitu. Questo legame, osservato da diversi studiosi, ${ }^{68}$ è chiaramente visibile da tre fonti principali.

59 Lex Orchia sumptuaria, 182 a.C.; Lex Fannia sumptuaria, 161 a.C.; Lex Licinia sumptuaria, 137-103 a.C.; Lex Cornelia sumptuaria, 81 a.C.; Lex Antia sumptuaria, 68 a.C.; Lex Iulia, 46 a.C.

$60 \quad$ Lex Orchia sumptuaria, 182 a.C.; Lex Fannia sumptuaria, 161 a.C.

61 Lex Fannia sumptuaria, 161 a.C.; Lex Licinia sumptuaria, 137-103 a.C.; Lex Aemilia sumptuaria, 115 a.C.; Lex Iulia, 46 a.C.

62 COUDRY, Loi et société, pp. 138-139.

63 Heracl. Lemb. Exc. Polit. 73. Cfr. COUDRY, Loi et société, p. 138.

64 Ath. Deipn. 6, 245c. Cfr. GALLO, L. Le leggi suntuarie greche e l'alimentazione. AION, 1993, Vol. 15, pp. 173-203; COUDRY, Loi et société, p. 13.

65 Plato, Leges VI, 775a.

66 Va menzionato il tentativo di GALLO, op. cit., pp. 173-203, di trovare un parallelo della legislazione romana dedicata al lusso del convivium, i cui risultati non sono largamente condivisi. Cfr. COUDRY, Loi et société, p. 138. A questo proposito Veyne (VEYNE, P. Le pain et le cirque: sociologie historique d'un pluralisme politique. Paris: Seuil, 1976, pp. 416-417) osserva che mentre nel mondo greco i banchetti dell'intera comunità sono un fenomeno comune, a Roma mancano occasioni pubbliche in cui i ceti oligarchici partecipino a banchetti insieme alla plebe. D'altro canto, i personaggi importanti offrono spesso banchetti ai ceti bassi, seguendo, però, un modello gerarchico verticale, tipico del rapporto clientelare.

67 Su questo problema importantissimo lo studio di VEYNE, op. cit. Egli sottolinea (pp. 416-417) che dei quattro tipi di atti evergetici noti a Roma, i.e. edifici, congiaria, spettacoli gladiatori e banchetti, gli ultimi due sono nati come cerimonie di famiglia e hanno dunque connotati più personali. Proprio in virtù di ciò l'organizzazione di munera o banchetti diventa uno dei meccanismi preferiti per la corruzione elettorale.

68 SAUERWEIN, op. cit., pp. 140-144; GRIFFIN, op . cit.; BALTRUSCH, op . cit., pp. 96-98; LINTOTT, A Electoral Bribery in the Roman Republic. JRS, 1990, Vol. 80, pp. 1-16, soprattutto pp. 5-6. NADIG, op. cit.; BOTTIGLIERI, op. cit., pp. 166-167; COUDRY, Loi et société, p. 156; COUDRY, M. Loi Antia sumptuaria. 
La prima è il trattato teorico Commentariolum Petitionis databile al 64 a.C. ${ }^{69}$ Tra i consigli per il candidato viene incluso quello di offrire banchetti o di fare in modo che i suoi amici ne offrano in onore suo.

Benignitas autem late patet. est in re familiari, quae quamquam ad multitudinem pervenire non potest, tamen ab amicis si laudatur, multitudini grata est; est in conviviis, quae fac et abs te et ab amicis tuis concelebrentur et passim et tributim; est etiam in opera, quam pervulga et communica, curaque ut aditus ad te diurni nocturnique pateant, neque solum foribus aedium tuarum sed etiam vultu ac fronte, quae est animi ianua; quae si significat voluntatem abditam esse ac retrusam, parvi refert patere ostium. homines enim non modo promitti sibi, praesertim quod de candidato petant, sed etiam large atque honorifice promitti volunt. ${ }^{70}$

Va notato, soprattutto, che questi convivia non sono riunioni di amici, neanche nel senso più ampio del termine, inteso come "partner politici". Anzi, poco prima del passo in questione l'autore sottolinea esplicitamente di aver esaurito i consigli su come stringere amicizie e di passare ai modi in cui assicurarsi il favore del popolo. ${ }^{71}$ È chiaro dunque che $\mathrm{i}$ banchetti, almeno in contesto elettorale, vengono intesi come riunioni di ampi gruppi di persone, legate poco o affatto al candidato con legami di amicitia o clientela.

La seconda fonte che collega espressamente il banchetto all'ambitus è la Lex Calpurnia de ambitu del 67 a.C. ${ }^{72}$ Essa vieta ai candidati di offrire banchetti volgo, ${ }^{73}$ i.e. a grandi gruppi di persone e anche di distribuire i posti per spettacoli gladiatori tributim. Si crede, ${ }^{74}$

69 Sul problema del suo autore, datazione e autenticità cfr. HENDERSON, M. I. De commentariolo petitionis. JRS, 1950, Vol. 40. No. 1-2, pp. 8-21; NISBET, R. G. M. The Commentariolum Petitionis: Some Arguments against Authenticity. JRS, 1061, Vol. 51, pp. 84-87; BALDSON, J. P. V. D. The Commentariolum Petitionis. CQ, 1963, Vol. 13, pp. 242-250; NARDO, D. Il Commentariolum Petitionis, La propaganda elettorale nella 'ars' di Quinto Cicerone. Padova: Liviana Editrice, 1970; MCDERMOTT, W. C. The Commentariolum Petitionis 2. Historia: Zeitschrift für Alte Geschichte, 1970, Vol. 19, pp. 384-385; RICHARDSON, J. S. The Commentariolum Petitionis. Historia: Zeitschrift für Alte Geschichte, 1971, Vol. 20, pp. 436-442; RAMSEY, J. A Reconstruction of Q. Gallius' Trial for "Ambitus": One Less Reason for Doubting the Authenticity of the Commentariolum Petitionis. Historia: Zeitschrift für Alte Geschichte, 1980, Vol.29, No. 4, pp. 402-421.

70 Q. Cic. Pet. 44.

71 Q. Cic. Pet. 41: quoniam de amicitiis constituendis satis dictum est, dicendum est de illa altera parte petitionis quae in populari ratione versatur. ea desiderat nomenclationem, blanditiam, adsiduitatem, benignitatem, rumorem, spem in re publica.

72 Cic. Balb. 57; Cic. Clu. 98; Cic. Corn. 1, fr. 25 P.; Cic. Corn. 1, fr. 40 P.; Cic. Corn. 1, fr. 41 P. Cic. Corn. 1, fr. 46 P. Cic. Mur. 46; Cic. Mur. 67; Cic. Or. tog. cand. fr. 14 P.; Dio 36, 38-39; Schol. Bob. 78 St; Ascon. 55 St.; Ascon. 68 St. Cfr. MCDONALD, W. The Tribunate of Cornelius. CQ , 1929, Vol. 23, pp. 199-205; ROTONDI, op. cit., p. 374; GRIFFIN, op. cit., pp. 196-203; LINTOTT, Electoral Bribery, pp. 1-16; DAVID, J.-M. - DONDIN, M. Dion Cassius XXXVI, 41, 1-2. Conduites symboliques et comportements exemplaires de Lucullus, Acilius Glabrio et Papirius Carbo (78 et 67 a. C.). MEFRA, 1980, Vol. 92, pp. 199-213; RAMSEY, op. cit., pp. 402-421; WALLINGA, T. Ambitus in the Roman Republic. RIDA, 1994, Vol. 41, pp. 414-442, soprattutto p. 426; GRUEN, E. The Last Generation of the Roman Republic. Berkeley: University of California Press, 1995, pp. 213-215; FERRARY, J.-L. Princeps legis et adscriptores: la collegialite des magistrats romains dans la procedure de proposition des lois. Revue de philologie, de littérature et d'histoire anciennes, 1996, Vol. 70, No. 2, pp. 217-246; FERRARY, J.-L. Loi Calpurnia Acilia de ambitu. In: Lepor. Leges Populi Romani, sous la dir. de Jean-Louis Ferrary et de Philippe Moreau. [online]. 5. 1. 2020 [cit. 15. 01. 2020]. Paris: IRHT-TELMA, 2007. Available at: http:// www.cn-telma.fr/lepor/notice1/.

73 Cic. Mur. 32, 67: ... si gladiatoribus volgo locus tributim et item prandia si volgo essent data, contra legem Calpurniam factum videri.

74 WALLINGA, Ambitus, pp. 414-442, soprattutto p. 426; FERRARY, Loi Calpurnia Acilia de ambitu. 
inoltre, che venisse vietata anche l'attività dei divisores, i personaggi che organizzano la corruzione elettorale diretta. ${ }^{75}$

La terza fonte, infine, è la summenzionata lex Antia sumptuaria del 64 a.C., secondo la quale a un magistrato o a un candidato viene vietato di attendere ripetutamente i banchetti, tranne da determinate categorie di persone. ${ }^{76}$

Tenendo presente queste tre fonti, sorge la domanda a proposito dei meccanismi pratici con i quali il banchetto elettorale potrebbe influenzare il voto.

Certamente, il meccanismo più facile da notare, e dunque più spesso sottolineato, è quello della "corruzione alimentare indiretta", esistente tutt'oggi e basata sulla gratitudine degli invitati per l'atto evergetico del pasto collettivo. ${ }^{77} \mathrm{Il}$ "dono del pasto" si potrebbe interpretare in chiave sociologica come un meccanismo per rafforzare i legami della clientela o per creare nuovi legami quasiclientelari, limitati solo alle elezioni concrete. ${ }^{78}$ A questo proposito si potrebbe ricordare anche l'ipotesi, espressa da numerosi studiosi, della votazione romana fondamentalmente apolitica che esprime piuttosto simpatie o interessi personali e non sostegno per una determinata corrente politica. ${ }^{79}$

Accanto al meccanismo dell'atto evergetico e la conseguente gratitudine, tuttavia, si possono intravvedere anche altri meccanismi più pratici per influenzare il voto attraverso $\mathrm{i}$ banchetti elettorali.

Va notato, in questo senso, il consiglio del Commentariolum Petitionis, secondo il quale il candidato deve organizzare banchetti passim e tributim. L'avverbio tributim appare nella lex Calpurnia de ambitu del 67 a.C. che proibisce di distribuire i posti per munera gladiatoria per tribù. ${ }^{80} \mathrm{Si}$ nota subito che nella lex Calpurnia non vengono vietati gli spettacoli in sé ma solo il raggruppamento del pubblico per unità elettorali. Questa particolarità pare in sintonia con il divieto dell'attività dei divisores nella stessa lex Calpurnia. ${ }^{81}$ È noto, infatti, che questi personaggi operavano a livello delle tribù, inizialmente distribuendo in modo del tutto legale i doni dei benefattori tra i membri della tribù, ma gradualmente trasformandosi in età tardorepubblicana in agenti della corruzione elettorale diretta. ${ }^{82}$

Si potrebbe, dunque, ipotizzare, che la proibizione dei divisores in un primo momento abbia portato a creare altri modi in cui organizzare i votanti per unità elettorale allo scopo di distribuire soldi o regali. Tra questi rientra indubbiamente il raggruppamento del pubblico dei giochi tributim, funzionale non solo alla corruzione elettorale indiretta attraverso

75 MOURITSEN, H. Plebs and Politics in the Late Roman Republic. Cambridge, Cambridge University Press, 2001, pp. 112-117; TAYLOR, L. R. - LINDERSKI, J. The voting districts of the Roman Republic: the thirty-five urban and rural tribes. Ann Arbor: University of Michigan Press, 2013, p. 15.

76 Aul. Gell. Noct. Att. 2, 24, 13; Lex deinde Antia praeter sumptum aeris id etiam sanxit, ut qui magistratus esset magistratumue capturus esset, ne quo ad cenam, nisi ad certas personas, itaret. Cfr. Supra.

77 VEYNE, op. cit., pp. 415-430.

78 LINTOTT, Electoral Bribery, pp. 5-6.

79 TAYLOR, L. R. Party Politics in the age of Caesar. Berkeley: University of California Press, 1964 MEIER, Ch. Res publica amissa: Eine Studie zu Verfassung und Geschichte der späten römischen Republik. Wiesbaden: Franz Steiner, 1966, pp. 191-196; VEYNE, op. cit., pp. 421-422.

80 Cfr. Cic. Mur. 32, 67: ... si gladiatoribus volgo locus tributim et item prandia si volgo essent data, contra legem Calpurniam factum videri.

81 Cic. Corn. 1, fr. 40 P.: (Repugnat) ut diuisores, quos honoris sui ministros esse uoluerat, lege ambitus uellet affligere.; Cic. Corn. 1, fr. 41 P.: Quare cum haec populus R. uideret et cum a tribunis pl. doceretur, nisi poena accessisset in diuisores, extingui ambitum nullo modo posse, legem hanc Corneli flagitabat, illam quae ex S. C.ferebatur repudiabat... Cfr. GRIFFIN, op. cit., p. 196.

82 Cfr. supra, MOURITSEN, op. cit., pp. 112-117; TAYLOR - LINDERSKI, op. cit., p. 15. 
l'offerta di un atto evergetico, ma anche alla continuazione della corruzione diretta. Dopo la lex Calpurnia anche questo diventa illegale, portando al consiglio di Quinto Cicerone di organizzare i banchetti elettorali tributim, forse anche in vista del facilitare la distribuzione di regali o soldi per tribù.

Se l'ipotesi della distribuzione di regali elettorali durante i banchetti fosse vera, si potrebbe offrire anche una possibile interpretazione del divieto della lex Fannia del 161 a.C. riguardo l'introduzione di più di 100 libbre di argento durante un banchetto. ${ }^{83}$ Da un punto di vista moralistico, esso viene spesso interpretato come limitante il lusso nel vasellame utilizzato durante il convivium. Tuttavia, si nota che la quantità dell'argento sembra così eccessiva per essere riferita solo al vasellame che alcuni credono che il testo sia corrotto. ${ }^{84}$ Il divieto di introdurre quantità eccessive d'argento, tuttavia, diventa più comprensibile se si pensa alla possibile distribuzione di regali durante il banchetto. Da notare, infatti, è che alcune epigrafi sembrano attestare la prassi di distribuire ai partecipanti ai banchetti elettorali delle coppette iscritte con il nome del candidato. ${ }^{85}$

Va ricordato, inoltre, che le leggi suntuarie sembrano avere come obiettivo, forse anche principale, quello di scoraggiare la riunione di grandi gruppi di persone in ambito privato. Interpretando questo divieto in chiave elettorale, si potrebbe trovare un parallelo anche con le proibizioni dell'attività dei sectatores dalla lex Fabia. ${ }^{86}$ Come sottolinea Quinto Cicerone, ${ }^{87}$ per la reputazione del candidato è di grande importanza la presenza di molte persone che lo accompagnino, sia per le strade (in qualità di sectatores), sia nella sua domus. La precisazione che la casa del candidato deve essere piena di notte rimanda chiaramente ai banchetti serali. Trovandoci in età tardorepubblicana, è impossibile non pensare alle bande di Clodio e Milone ed è dunque facile comprendere perché la concentrazione di molte

83 Aul. Gell. Noct. Att. 2, 24, 2: ... neque argenti in conuiuio plus pondo quam libras centum inlaturos.

84 ROSIVACH, op. cit., pp. 3-4.

85 Si tratta, in questi casi, di coppette in argilla di poco valore; non pare improbabile, tuttavia, pensare alla distribuzione anche di vasellame più costoso. Cfr. PANCIERA, S. Catilina e Catone su due coppette romane.

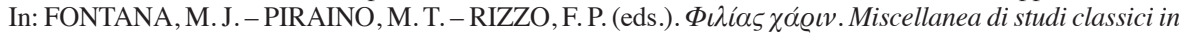
onore di Eugenio Manni. Vol. VI. Roma: Bretschneider, 1980, pp. 1635-1663; DENIAUX, E. De l'ambitio à l'ambitus: les lieux de la propagande et de la corruption électorale à la fin de la République. In: L'Urbs: espace urbain et histoire (Ier siècle av. J.-C. - IIIe siècle ap. J.-C.). Actes du colloque international de Rome (8-12 mai 1985). Roma: École Française de Rome, 1987, pp. 279-304; PANCIERA, S. Catilina e Catone candidati. In: PANCIERA, S. Epigrafi, epigrafia, epigrafisti. Scritti vari editi e inediti (1956-2005) con note complementari e indici. Roma: Quasar, 2006. Vol. II., pp. 1059-1072.

86 Cic. Mur. 71: Itaque et legi Fabiae quae est de numero sectatorum, et senatus consulto quod est L. Caesare consule factum restiterunt. Cfr. Plut. Cat. mi. 8, 4-5. ROTONDI, op. cit., pp. 378-379; KINSEY, T. E. Cicero, Pro Murena 71. RBPh, 1965, Vol. 43, pp. 57-59; GRUEN, The Last Generation, p. 216; WALLINGA, Ambitus, pp. 414-442; FERRARY, J. L. Loi Fabia. In: Lepor. Leges Populi Romani, sous la dir. de Jean-Louis Ferrary et de Philippe Moreau. [online]. 28. 11. 2014 [cit. 19. 11. 2019]. Paris: IRHTTELMA, 2007. Available at: http://www.cn-telma.fr/lepor/notice376/.

87 Q. Cic. Pet. 50: sequitur enim ut de rumore dicendum sit, cui maxime serviendum est. sed quae dicta sunt omni superiore oratione, eadem ad rumorem concelebrandum valent, dicendi laus, studia publicanorum et equestris ordinis, hominum nobilium voluntas, adulescentulorum frequentia, eorum qui abs te defensi sunt adsiduitas, ex municipiis multitudo eorum quos tua causa venisse appareat, bene te ut homines nosse, comiter appellare, adsidue diligenter petere, benignum ac liberalem esse et loquantur et existiment, domus ut multa nocte compleatur, omnium generum frequentia adsit, satis fiat oratione omnibus, re operaque multis; perficiatur id quod fieri potest labore et arte ac diligentia, non ut ad populum ab his omnibus fama perveniat sed ut in his studiis populus ipse versetur. 
persone, riunite da legami sociali o politici, possa essere indesiderabile in un periodo di instabilità politica. ${ }^{88}$

Infine, in vista del legame tra le leges sumptuarie e le campagne elettorali, conviene osservare un importante parallelo tra le disposizioni di due leggi. La prima è la summenzionata lex Antia sumptuaria del 68 a.C. che proibisce al candidato di partecipare ripetutamente a banchetti, tranne se organizzati da certe persone. Il provvedimento serve a prevenire appunto la prassi di usare amici come evergeti sostitutivi per evitare l'accusa di ambitus, attestata e raccomandata anche da Quinto Cicerone. ${ }^{89}$

La seconda legge che va osservata è la Lex coloniae Iuliae Genetivae (Lex Ursonensis $)^{90}$ degli anni 40 a.C. Nonostante si tratti di una legge municipale provinciale, è universalmente accettata l'idea che essa riproduce modelli legislativi urbani. Essa proibisce al candidato di offrire banchetti elettorali ma anche di partecipare a banchetti, organizzati da evergeti sostitutivi.

Certamente, se il candidato violasse questi divieti, egli potrebbe essere accusato di ambitus. L'accusa di ambitus, tuttavia, difficilmente potrebbe colpire gli evergeti sostitutivi, in quanto solo indirettamente legati alla campagna elettorale. D'altronde, sarebbe ragionevole supporre che tali evergeti sostitutivi potessero essere scoraggiati, anche se indirettamente, dalla legislazione suntuaria che vieta di organizzare grandi banchetti con molti invitati, a prescindere se l'organizzatore sia coinvolto direttamente nelle elezioni o meno.

Qualche parola, infine, a proposito di una della possibili motivazioni che hanno portato alla creazione della serie di leges sumptuariae. L'opinione dominante degli studiosi collega la legislazione suntuaria con la forte competitività tra gli esponenti oligarchici e la forte pressione sociale sui ceti alti di dimostrare il loro status, al punto di rischiare la bancarotta. ${ }^{91}$ Le leggi limitanti il lusso sarebbero, in quest'ottica, un mezzo per proteggere il patrimonio dell'élite dall'irragionevole gara per status sociale.

Tuttavia, se si considera il possibile legame tra le leggi limitanti i banchetti e le campagne elettorali, è possibile individuare anche un'ulteriore motivazione. Si potrebbe intravvedere un tentativo dei membri dell'élite di prevenire la possibilità che i personaggi più

88 Sull'uso di violenza durante le campagne elettorali cfr. LINTOTT, A. Violence in Republican Rome. Oxford: Clarendon Press, 1968, soprattutto pp. 67-89; VEYNE, op. cit., pp. 421, 510, n. 176.

89 Q. Cic. Pet. 44: ... in conviviis, quae fac et abs te et ab amicis tuis concelebrentur.

90 CIL II, 5, 1022, 132: ne quis in c(olonia) G(enetiua) post h(anc) l(egem) datam petitor kandidatus,/ quicumque in c(olonia) G(enetiua) I(ulia) mag(istratum) petet, magistratus\{ue\} peten-/ I16/ di causa in eo anno, quo quisque anno petitor/ kandidatus mag(istratum) petet petiturusue erit, mag(istratus) pe-/tendi $<$ c(ausa)> conuiuia facito neue at cenam quem\{ue\}/ иocato neue conuiuium habeto neue facito sc(iens) $<d($ olo $)>m($ alo $)$, / I20l quo qui $<s>$ suae petitionis causa conui $<$ ui $>$ um habeat/ ad cenamue quem $\{$ ue\} uocet, praeterdum quod ip-/se kandidatus petitor in eo anno, <quo> mag(istratum) petat,/ uocari[t] dumtaxat in dies sing(ulos) h(omines) (nouem) conui<ui $>$ um/ 241 hab $<$ uerit $>$, si uolet, s(ine) d(olo) $m(a l o)$. neue quis petitor kandidatus/ donum munus aliudue quit det largiatur peti-/tionis causa sc(iens) $d($ olo) m(alo). neue quis alterius petitionis/ causa conuiuia facito neue quem ad cenam uoca-/ I28/ to neue conuiuium habeto, neue quis alterius pe-/titionis causa cui quit d[on]um munus aliutue quit/ dato donato largito sc(iens) d(olo) m(alo). si quis atuersus eal fecerit, (sestertium) (quinque milia) c(olonis) c(oloniae) G(enetiuae) I(uliae) d(are) d(amnas) e(sto), eiusque pecuniae cui eor(um)/ I32I uolet rec(iperatorio) iudic(io) aput IIuir(um) praef(ectum)<ue > actio petitio per-/sec(utio)que ex h(ac) l(ege) i(us) potest(as) que esto. vacat/. Cfr. CRAWFORD, M. Roman Statutes. Vol. I. London: Institute of Classical Studies, University of London, 1996, pp. 393-454, con ampia bibliografia.

91 DAUBE, D. Roman law. Edinburgh: Edinburgh Univ. Press, 1969, pp. 117-128; CRAWFORD, M. The Roman Republic. Cambridge, Mass.: Harvard University Press, 1993, p. 76; WALLINGA, Ambitus, p. 439. 
abbienti di questa élite dominino la scena politica in virtù della loro ricchezza eccessiva. Le leges sumptuariae servirebbero, dunque, anche a privare (o almeno a limitare) alcuni dei concorrenti nella lotta politica dell'uso di un potentissimo mezzo - l'atto evergetico di offrire un pasto collettivo. 